\title{
Effective stakeholder engagement: design and implementation of a clinical trial (SWOG S1415CD) to improve cancer care
}

Sarah Barger ${ }^{1}$, Sean D. Sullivan ${ }^{2 *}$, Ari Bell-Brown ${ }^{1}$, Brad Bott $^{3}$, Anne Marie Ciccarella ${ }^{4}$, John Golenski ${ }^{5}$, Mark Gorman ${ }^{6}$, Judy Johnson ${ }^{7}$, Karma Kreizenbeck ${ }^{1}$, Florence Kurttila ${ }^{8}$, Ginny Mason', Jamie Myers ${ }^{10}$, Carole Seigel ${ }^{11}$, James L. Wade $1 I^{12}$, Guneet Walia ${ }^{13}$, Kate Watabayashi ${ }^{1}$, Gary H. Lyman ${ }^{1}$ and Scott D. Ramsey ${ }^{1}$

\begin{abstract}
Background: The Fred Hutchinson Cancer Research Center has engaged an External Stakeholder Advisory Group (ESAG) in the planning and implementation of the TrACER Study (S1415CD), a five-year pragmatic clinical trial assessing the effectiveness of a guideline-based colony stimulating factor standing order intervention. The trial is being conducted by SWOG through the National Cancer Institute Community Oncology Research Program in 45 clinics. The ESAG includes ten patient partners, two payers, two pharmacists, two guideline experts, four providers and one medical ethicist. This manuscript describes the ESAG's role and impact on the trial.
\end{abstract}

Methods: During early trial development, the research team assembled the ESAG to inform plans for each phase of the trial. ESAG members provide feedback and engage in problem solving to improve trial implementation. Each year, members participate in one in-person meeting, web conferences and targeted email discussion. Additionally, they complete a survey that assesses their satisfaction with communication and collaboration. The research team collected and reviewed stakeholder input from 2014 to 2018 for impact on the trial.

Results: The ESAG has informed trial design, implementation and dissemination planning. The group advised the trial's endpoints, regimen list and development of cohort and usual care arms. Based on ESAG input, the research team enhanced patient surveys and added pharmacy-related questions to the component application to assess order entry systems. ESAG patient partners collaborated with the research team to develop a patient brochure and study summary for clinic staff. In addition to identifying recruitment strategies and patient-oriented platforms for publicly sharing results, ESAG members participated as co-authors on this manuscript and a conference poster presentation highlighting stakeholder influence on the trial. The annual satisfaction survey results suggest that ESAG members were satisfied with the methods, frequency and target areas of their engagement in the trial during project years 1-3.

Conclusions: Diverse stakeholder engagement has been essential in optimizing the design, implementation and planned dissemination of the TrACER Study. The lessons described in the manuscript may assist others to effectively partner with stakeholders on clinical research.

Keywords: Stakeholder engagement, Patient engagement, Oncology, Clinical trial, Cancer, Cancer care delivery research

\footnotetext{
*Correspondence: sdsull@u.washington.edu; sdsull@uw.edu

${ }^{2} \mathrm{CHOICE}$ Institute, School of Pharmacy, University of Washington, Seattle, WA

98195, USA

Full list of author information is available at the end of the article
}

(c) The Author(s). 2019 Open Access This article is distributed under the terms of the Creative Commons Attribution 4.0 International License (http://creativecommons.org/licenses/by/4.0/), which permits unrestricted use, distribution, and reproduction in any medium, provided you give appropriate credit to the original author(s) and the source, provide a link to the Creative Commons license, and indicate if changes were made. The Creative Commons Public Domain Dedication waiver (http://creativecommons.org/publicdomain/zero/1.0/) applies to the data made available in this article, unless otherwise stated. 


\section{Background}

Recognition of the value and importance of integrating stakeholder engagement into comparative effectiveness research (CER) and patient-centered outcomes research (PCOR) to improve health care delivery continues to grow [1, 2]. Stakeholder engagement in research is defined as an iterative process of actively soliciting the knowledge, experience, judgment and values of individuals selected to represent a broad range of interests in a particular issue, for the dual purposes of creating a shared understanding and making relevant, transparent and effective decisions [1]. Patients and other relevant stakeholders can support the objectives of CER and PCOR by contributing to evidence-based, patient-centered health interventions and tools that help patients and consumers make informed health care decisions [3]. Studies have found that engaging stakeholders in the development and execution of CER studies influenced research prioritization [4-6], provided valuable insights regarding study design [7, 8] and data analysis [9], and led to greater relevance and uptake of research results $[10,11]$. In particular, patients may enhance CER and PCOR by providing experiential knowledge to a research question or study design, informing data collection and analysis, reviewing and interpreting results, and improving translation and dissemination of key findings [12-14].

Despite the potential benefits, there are significant challenges in embedding stakeholders into the research continuum. Consistent engagement requires a significant investment of time and resources from both researchers and stakeholders $[13,15]$. Deverka and colleagues evaluated stakeholder perceptions of engagement in CER and identified barriers including unclear role expectations, limited opportunities to communicate with clinical investigators, and difficulty in balancing timely input from stakeholders with the pace of research needs [16]. At present, no general agreement exists regarding best practices of longitudinal stakeholder engagement in CER $[1,10]$. In oncology, there is experience with multistakeholder engagement in CER trial design [17-19] but no experience with ongoing engagement from design through trial implementation and dissemination. Accordingly, the purpose of this manuscript is to describe one model for longitudinal stakeholder engagement that has been effectively implemented to support a pragmatic clinical trial in cancer care delivery.

Known as TrACER (A Pragmatic Trial Assessing CSF Prescribing Effectiveness and Risk), this randomized, controlled CER study evaluates a guideline-based, automated order entry system for prescribing primary prophylactic colony stimulating factors (CSF) against usual care (i.e. physician-directed CSF prescribing) for patients receiving myelosuppressive chemotherapy [17].
TrACER is one of 23 studies in the PCORI Pragmatic Clinical Studies Program [20] and is being conducted by SWOG through the National Cancer Institute Community Oncology Research Program (NCORP) network in 45 clinics that support cancer care delivery research. The primary goals of the trial are to determine the impact of the order entry system on CSF prescribing, overall rates of febrile neutropenia (FN) and the rate of FN among patients receiving intermediate risk chemotherapy. Secondary objectives focus on the impact of the intervention on patient-centered outcomes, such as health-related quality of life and knowledge of the benefits and risks of CSF. The TrACER Study attempts to answer questions that are important to patients and healthcare systems and therefore involves a variety of stakeholders in the design, implementation and dissemination of results.

This manuscript describes the process and resultant impact of engaging a large group of stakeholders in the planning and implementation of the TrACER Study. Our approach and lessons learned contribute to the growing evidence of the value of stakeholder engagement in CER, specifically in oncology.

\section{Methods}

\section{Initial stakeholder engagement in TrACER}

Prior to submitting a proposal for funding, the research team assembled an External Stakeholder Advisory Group (ESAG) to inform each phase of the trial from planning and design to implementation and dissemination. The ESAG is comprised of 21 national leaders from 14 different states and territories in the United States, including ten patient partners, two payers, two pharmacists, two guideline experts, four providers (nursing, medical oncology, internal medicine) and one medical ethicist. Four patient partners joined the ESAG during the proposal planning phase, and six additional patient partners from the SWOG Patient Advocate Committee [21] joined the group one year into the trial. All patient partners had prior experiences working with cancer care professionals and/or researchers and navigating the health care system in their roles as patient advocates, research partners, patients/survivors and caregivers. In alignment with PCORI's compensation framework [22], patient partners received financial compensation for their time and effort as study advisors and were reimbursed for all costs related to travel to ESAG meetings.

\section{Development of engagement plan}

During the planning phase, the research team developed a four-year engagement plan outlining the aims of stakeholder engagement, roles and responsibilities of ESAG members and their expected activities throughout the trial. As demonstrated in Fig. 1, stakeholders are embedded in the lifecycle of the clinical trial, from design and protocol 


\section{Year 1}

- Refine study protocols; for example, translate any non-specific guideline language into more specific standing orders and other clinical direction

- Provide guidance on strategies to implement standing orders within participating SWOG clinics

- Identify strategies to recruit clinics

\section{Years 2 and 3}

- Respond to issues with patient recruitment, retention, use of standing orders and any other study implementation issues

- Publicize the study more broadly among stakeholder groups

- Develop ideas for dissemination, implementation and knowledge transition of study findings once results are available

\section{Year 4}

- Refine strategies for effective dissemination and translation of study results and/or clinical guidelines into clinical practice, building on discussions from earlier meetings

- Recommend coverage and payment policies to encourage uptake of standing CSF order systems and other practice improvement strategies based on study results

Fig. 1 ESAG Activities According to Engagement Plan (H: 3.53", W: 6.39")

development through interpretation and dissemination of results.

\section{Structure and facilitation of ESAG meetings and communication}

Each year, the stakeholders and research team convene during two web conferences, two patient partner-specific web conferences and one in-person meeting held in conjunction with the SWOG Group Meeting. These interactive meetings facilitate regular communication about trial progress, open discussion and collaborative problem solving. During patient-specific meetings, the research team provides a study briefing with an explanation of any relevant clinical or statistical concepts that may be unfamiliar; in addition, the group focuses on patientspecific topics like barriers to patient recruitment. The team reaches out to specific stakeholders over email to request feedback on issues related to their areas of expertise. For example, guideline experts and pharmacists on the ESAG provided input over email during the review of the FN risk algorithm in 2015 and 2017 to ensure that the algorithm reflects up-to-date guidelines from the National Comprehensive Cancer Network $(\mathrm{NCCN})$. To ensure timely decision-making regarding study issues, stakeholders offer their recommendations during a two-week comment period. The research team discusses feasibility of ESAG suggestions, determines a course of action, and reports back to group on the final decisions made.

\section{Annual stakeholder evaluation and reporting}

The research team evaluates stakeholder engagement, satisfaction and impact on the trial annually through the ESAG Feedback Survey. The 21-question survey addresses communication, meeting structure, involvement and input, respect and value, and ESAG areas of interest for the next project year. Comment boxes provide an opportunity for additional feedback or suggestions. Stakeholders complete the survey prior to the annual in-person ESAG meeting so that the research team can share the results with the ESAG and discuss the next year's engagement activities.

\section{Results}

The research team solicits and integrates ESAG feedback into the trial during each phase. Below and in Fig. 2, we describe how ESAG feedback has impacted trial design, implementation, recruitment and early dissemination planning.

\section{Impact on trial design Proposal planning}

During the proposal planning phase, the ESAG advised the research team to adjust trial endpoints and base the standing order intervention solely on chemotherapy regimen risk, not individual patient characteristics such as comorbidities. In addition, the group raised concerns about only consenting patients in the intervention arms, so the research team extended the consent process to 


\begin{tabular}{|c|c|c|c|}
\hline Impact on Trial Design & $\begin{array}{l}\text { Impact on Trial } \\
\text { Implementation }\end{array}$ & Impact on Recruitment & $\begin{array}{c}\text { Impact on Early } \\
\text { Dissemination Planning }\end{array}$ \\
\hline $\begin{array}{l}\text { - Revised study endpoints } \\
\text { - Advised FN risk algorithm } \\
\text { and regularly reviewed } \\
\text { regimens and FN risk levels } \\
\text { - Advised development of } \\
\text { cohort and usual care arms } \\
\text { - Added questions to patient } \\
\text { surveys to capture: } \\
\text { - Out-of-pocket cost of CSF } \\
\text { - Socioeconomic status } \\
\text { - Survey completion by } \\
\text { caregiver } \\
\text { - Reason for declining CSF } \\
\text { - Donation of leftover CSF } \\
\text { doses } \\
\text { - Added pharmacy-related } \\
\text { questions to component } \\
\text { application that assesses } \\
\text { order entry system } \\
\text { - Reviewed a statement } \\
\text { clarifying that study is not } \\
\text { endorsing CSF; advised on } \\
\text { dissemination to clinics }\end{array}$ & $\begin{array}{l}\text { - Co-developed patient } \\
\text { brochure with research team } \\
\text { - Enhanced study summary } \\
\text { called TrACER Talking Points } \\
\text { by recommending: } \\
\text { - Lay language about trial } \\
\text { - Inclusion of a diagram to } \\
\text { briefly describe study arms } \\
\text { - Compilation of financial } \\
\text { resources for patients, } \\
\text { given the expensive cost of } \\
\text { CSF }\end{array}$ & $\begin{array}{l}\text { - Advised strategies for } \\
\text { patient approach, including: } \\
\text { - Approach patients during } \\
\text { chemotherapy teaching } \\
\text { session or call prior to first } \\
\text { day of treatment } \\
\text { - Consider that patients } \\
\text { may feel overwhelmed, } \\
\text { anxious, or overloaded } \\
\text { with information } \\
\text { - Ensure that patients feel } \\
\text { welcomed and cared for } \\
\text { when approaching them }\end{array}$ & $\begin{array}{l}\text { - Served as co-authors on } \\
\text { manuscript and a conference } \\
\text { poster presentation } \\
\text { - Identified strategies for } \\
\text { broad dissemination of study } \\
\text { results, including: } \\
\text { - Scientific and health } \\
\text { journals } \\
\text { - Partnerships with national } \\
\text { non-profit organizations } \\
\text { (e.g., newsletters, } \\
\text { websites) } \\
\text { - Radio and podcast } \\
\text { coverage } \\
\text { - Communication } \\
\text { mechanisms of } \\
\text { participating hospitals } \\
\text { - Social media (e.g., Twitter, } \\
\text { Facebook, patient blogs) } \\
\text { and collaboration with } \\
\text { SWOG Digital Engagement } \\
\text { Committee }\end{array}$ \\
\hline
\end{tabular}

participants in all arms. ESAG members recommended that the research team track oral antibiotic use and clarify that physicians can override standing orders and prescribe CSF based on clinical judgment. These suggestions were incorporated into the protocol.

\section{Site feasibility survey}

When giving feedback on the trial design, the ESAG recognized the importance of measuring and characterizing usual care, given the heterogeneity of existing prescribing patterns. To better understand CSF prescribing patterns, the research team decided to survey clinic practices, conduct a retrospective audit of each practice's patterns for the 6 months prior to the trial, and add a parallel observational cohort study of practices ineligible for the primary randomization. In addition, the ESAG and research team developed a site feasibility survey to help assess clinic eligibility for the trial and understand practice-level characteristics as they pertain to prescribing CSF.

\section{Patient surveys}

ESAG patient partners made several recommendations for improving the trial's baseline and follow-up patient surveys. They emphasized the importance of tracking cost of CSF, which led the research team to add a question capturing out-of-pocket costs of CSF. In addition, they noted that patients sometimes receive CSF "donations" from other patients due to its high cost, so a question was added to capture if patients had any remaining doses of CSF, how much they had, and what they did with the remaining doses. Questions also were added to capture socioeconomic status (i.e. annual household income), who completed the survey (including a caregiver option) and the patient's understanding of his/ her risk of febrile neutropenia.

\section{Protocol and consent}

The ESAG refined numerous protocol areas, including site recruitment, data collection, trial design and randomization, order entry intervention and criteria for evaluation and endpoint analysis. Nurses and patient partners provided several suggestions to improve clarity of the consent forms for participants.

\section{FN risk algorithm}

A febrile neutropenia (FN) regimen-based risk algorithm based on current NCCN guidelines and peer-reviewed studies was developed by the research team with feedback from pharmacy and guideline experts in the ESAG. Each year when new NCCN guidelines are released, this risk algorithm is reviewed and updated by the same stakeholders.

\section{Impact on trial implementation TrACER patient brochures}

The ESAG suggested that it would be helpful to give patients a handout explaining the trial in lay terms. 
During an eight-month process, the patient partners collaborated with the research team to create a trifold brochure in both English and Spanish that describes the trial in a clear, understandable manner. Patient partners assisted in replacing medical jargon with non-technical language and articulating the expectations of patient participation, as well as risks and benefits. Study site staff reported that the brochure was highly valued as a simple tool for explaining the trial to eligible patients and a helpful summary document for clinic staff and providers.

\section{TrACER talking points}

In August 2016, study coordinators at participating sites recommended creating a summary handout to assist in answering any patient questions about the trial. In collaboration with ESAG patient partners, clinic coordinators, and the TrACER SWOG data coordinators, the research team developed TrACER Talking Points. This document includes a trial overview for investigators and patients, as well as a clinic group diagram with a brief overview of the different study arms.

\section{Physician statement}

Based on physician concerns regarding "mandated" CSF use, the research team developed a statement to clarify that the protocol-indicated standing orders should not be viewed as a clinical endorsement for the inclusion or exclusion of CSF in the chemotherapy order. Physicians on the ESAG reviewed this language and suggested that the team disseminate a shorter statement via the study newsletter and a longer statement for the study website, which was implemented accordingly.

\section{Impact on recruitment}

\section{Patient approach, recruitment and consent}

The TrACER Study opened to accrual in October 2016. The patient partner and ESAG web conferences in Spring 2017 focused on barriers to patient accrual, specifically the patient approach. To participate in the trial, patients must be consented prior to their first cycle of chemotherapy. Thus, ESAG members compiled a list of strategies for approaching patients during this narrow timeframe, and this information was shared with sites that struggled to accrue patients. ESAG feedback also was incorporated into the trial's frequently asked questions document available for all sites via the SWOG/ Cancer Trials Support Unit study website.

\section{Impact on dissemination and future directions}

From the beginning of the trial, the ESAG has been vocal about the importance of disseminating results to all stakeholders using multiple mediums. During one web conference, the group identified various methods for dissemination of trial results, including social media (i.e. patient blogs, Facebook, Twitter), publications such as CURE magazine, newsletters of national advocacy organizations and radio talk shows. They encouraged the research team to incorporate results into pertinent guidelines (e.g., NCCN, American Society of Clinical Oncology) and to publish findings, both positive and unsuccessful results, in open access academic journals for the learning value. In addition, patient partners emphasized the importance of conveying results to participants and their caregivers/families and provided insights into appropriate vehicles for achieving this communication that will not burden clinical trial investigators.

During project year 3 , the patient partners of the ESAG served as co-authors on a poster detailing the influence of patient engagement on trial design and implementation that was presented at a national quality conference. Importantly, ESAG members contributed to the development of this manuscript's structure and content through planning calls and email communication over the course of one year. During year 4, the ESAG will continue advising the study team on patient enrollment and dissemination planning. In addition, ESAG members will review and provide feedback on reports from the Data and Safety Monitoring Committee, which include recommendations for changing the study based on interim analyses.

\section{Discussion}

ESAG members have played a significant role in optimizing the design, implementation, recruitment, and early dissemination planning of the TrACER Study. The group's diverse perspectives and experiences have enriched group discussion about the trial and catalyzed real-time solutions to challenges over the course of three years. For example, patient partners have drawn on their varied experiences as clinical trial participants, chemotherapy patients, study coordinators, caregivers and patient advocates to contextualize challenges around patient recruitment and propose effective strategies to overcome these challenges. Guideline experts, providers and pharmacists have contributed invaluable clinical knowledge about CSF prescribing and ensured that the research team is responsive to frequently changing guidelines. Our medical ethicist, payers and patient partners have initiated important discussions about the high cost of CSF and the resultant impact on patients, particularly those involved in the trial. In sum, the continuous and strategic involvement of ESAG members in the trial facilitated critical thinking, sharing of unique insights, and collaborative problem solving, which led to key improvements in trial design and implementation.

Effective stakeholder engagement in the TrACER Study can be attributed to numerous factors. According 


\section{Best Practices of Stakeholder Engagement in Clinical Research}

\section{Early and Continual Involvement of Stakeholders}

- Engage stakeholders throughout clinical trial lifecycle from design to dissemination

- Identify specific challenges to discuss during meetings

- Share study information regularly with stakeholders

\section{Personalized Approach to Engagement}

- Arrange meetings in advance according to stakeholder availability

- Utilize multiple communication methods (i.e. email, web conference, in-person meeting) to create flexibility in participation

\section{Transparency in Decision-Making}

- Explain why study decisions were made and the impact of each decision upon implementation

- Demonstrate to stakeholders how their input has influenced the trial

\section{Open and Honest Communication to Foster Meaningful Collaboration}

- Set expectations of mutual trust and respect in communication

- Encourage sharing of ideas and open dialogue throughout meetings

\section{Clear Explanation of Study Topics}

- Explain study concepts and challenges in clear, understandable terms to ensure all stakeholders can fully participate in discussion

- Empower stakeholders to educate one another and the research team on study-related topics

Fig. 3 Best Practices of Stakeholder Engagement (H: 3.88", W: 6")

to the results from the annual satisfaction survey completed in 2016 and 2017, ESAG members were satisfied with the methods, frequency and target areas of their engagement in the trial during project years 1 and 2. Across both years, ESAG members favored regular updates on recruitment and enrollment, as well as participation in discussions with the research team about implementation challenges and ways to troubleshoot issues in the trial. They also preferred practical, relevant communication about time-sensitive issues so that they could be "nimble and pivot as necessary" as one ESAG member said. Interestingly, ESAG members ranked in-person meetings as the most effective method for receiving information and providing input during project year 2 , whereas they ranked in-person meetings as the least effective option in project year 1 . However, receiving information via webinar was consistently a preferred option during both project years.

During year 3, ESAG members and the research team compiled a list of best practices of stakeholder engagement (Fig. 3) to help guide other research groups interested in incorporating stakeholder engagement into health care delivery research and clinical trials.

\section{Conclusions}

Effective stakeholder engagement in the design, conduct and reporting of clinical research is essential to project success, particularly in cancer care delivery research. We have described the approach by which we engaged a diverse set of stakeholders for a pragmatic clinical trial testing a method to improve the appropriate use of an expensive supportive therapy for cancer. Our experience illustrates how different perspectives can lead to improved, patient-centric design and facilitate implementation of a trial. The lessons described in the manuscript may assist others with interest in effective use of stakeholders to inform clinical research.

\footnotetext{
Abbreviations

ASCO: American Society of Clinical Oncology; CER: Comparative effectiveness research; CSF: Colony stimulating factors; ESAG: External Stakeholder Advisory Group; FN: Febrile neutropenia; NCCN: National Comprehensive Cancer Network; NCORP: National Cancer Institute Community Oncology Research Program; PCOR: Patient-centered outcomes research; PCORI: PatientCentered Outcomes Research Institute; SWOG: Formerly the "Southwest
} 
Oncology Group," now called SWOG Cancer Research Network; TrACER: Trial Assessing CSF Prescribing Effectiveness and Risk

\section{Acknowledgements}

The research team would like to acknowledge the External Stakeholder Advisory Group (ESAG) for its impact on the TrACER Study (SWOG S1415CD).

\section{Disclaimer}

The content is solely the responsibility of the authors and does not necessarily represent the official views of the National Institutes of Health. The views presented in this work are solely the responsibility of the authors and do not necessarily represent the views of the Patient-Centered Outcomes Research Institute (PCORI), its Board of Governors or Methodology Committee.

\section{Authors' contributions}

All authors collaboratively developed the conceptual framework for the manuscript. SB conducted the literature review and drafted the manuscript. SDS, SDR, ABB and GHL edited the draft for clarity and contributed to analysis, discussion and future directions. BB, AMC, JG, MG, JJ, FK, CS, WG, GM, JLW, JM, GW, KK and KW reviewed the draft and contributed to the interpretation of results and discussion section. ABB assisted in manuscript preparation. All authors have read and approved the final manuscript.

\section{Funding}

Research reported in this manuscript was partially funded by the National Cancer Institute of the National Institutes of Health under award number 5UG1CA189974 and through a Patient-Centered Outcomes Research Institute (PCORI) award (PCS-1402-09988). The funding sources had no role in the design of the study and collection, analysis, and interpretation of data and in preparing, reviewing and submitting the manuscript.

\section{Availability of data and materials}

Not applicable.

\section{Ethics approval and consent to participate}

This study was approved by the Fred Hutchinson Cancer Research Center Institutional Review Board (IRB) under expedited review (IRB file \#: 8428; Assurance \# FWA00001920; Reviewing Committee D (9831)). Study participants signed a written informed consent to take part in this study.

\section{Consent for publication}

Not applicable.

\section{Competing interests}

The authors declare that they have no competing interests.

\section{Author details}

${ }^{1}$ Hutchinson Institute for Cancer Outcomes Research, Seattle, WA, USA. ${ }^{2} \mathrm{CHOICE}$ Institute, School of Pharmacy, University of Washington, Seattle, WA 98195, USA. ${ }^{3}$ Intermountain Healthcare, Salt Lake City, UT, USA. ${ }^{4}$ Independent Patient Research Partner and SWOG Digital Engagement Committee Member, New York, NY, USA. ${ }^{5}$ Kairoi Healthcare Strategies, San Francisco, CA, USA. ${ }^{6}$ Cancer Survivor Advisor, Silver Spring, MD, USA. ${ }^{7}$ SWOG Lung Committee Patient Advocate, St. Louis, MO, USA. ${ }^{8}$ SWOG GI Committee Patient Advocate, Citrus Heights, CA, USA. ' SWOG Breast Committee Patient Advocate, West Lafayette, IN, USA. ${ }^{10}$ University of Kansas, School of Nursing, Kansas City, KS, USA. ${ }^{11}$ SWOG GI (Pancreatic Cancer) Committee, Patient Advocate, Boston, MA, USA. ${ }^{12}$ Heartland NCORP, Decatur, IL, USA.

${ }^{13}$ Genentech, Inc, South San Francisco, CA, USA.

Received: 26 November 2018 Accepted: 4 June 2019

Published online: 11 June 2019

\section{References}

1. Deverka PA, Lavallee DC, Desai PJ, Esmail LC, Ramsey SD, Veenstra DL, et al. Stakeholder participation in comparative effectiveness research: defining a framework for effective engagement. J Comp Eff Res. 2012;1(2):181-94.

2. Hoffman A, Montgomery R, Aubry W, Tunis SR. How best to engage patients, doctors, and other stakeholders in designing comparative effectiveness studies. Health Aff. 2010;29(10):1834-41.
3. Gabriel SE, Normand S-LT. Getting the methods right - the Foundation of Patient-Centered Outcomes Research. N Engl J Med. 2012;367(9):787-90.

4. Esmail LC, Roth J, Rangarao S, Carlson JJ, Thariani R, Ramsey SD, et al. Getting our priorities straight: a novel framework for stakeholder-informed prioritization of cancer genomics research. Genetics In Medicine. 2013;15(2):115-22

5. Greenberg CC, Wind JK, Chang GJ, Chen RC, Schrag D. Stakeholder engagement for comparative effectiveness research in cancer care: experience of the DEcIDE Cancer consortium. J Comp Eff Res. 2013;2(2):117-25.

6. Filippou P, Smith AB. Prioritizing the patient voice in the development of urologic oncology research. Urol Oncol. 2017;35(9):548-51.

7. Abe J, Lobo JM, Trifiletti DM, Showalter TN. Providing guidance for genomics-based cancer treatment decisions: insights from stakeholder engagement for post-prostatectomy radiation therapy. BMC Med Inform Decis Mak. 2017;17(1):128.

8. Enzinger AC, Wind JK, Frank E, McCleary NJ, Porter L, Cushing H, et al. A stakeholder-driven approach to improve the informed consent process for palliative care. Patient Educ Couns. 2017;100(8):1527-36.

9. Klein G, Gold LS, Sullivan SS, Buist DSM, Ramsey SD, Kreizenbeck K, et al. Prioritizing comparative effectiveness research for cancer diagnostics using a regional stakeholder approach. J Comp Eff Res. 2012;1(3):241-55.

10. Concannon TW, Fuster M, Saunders T, Patel K, Wong JB, Leslie LK, et al. A systematic review of stakeholder engagement in comparative effectiveness and patient-centered outcomes research. J Gen Intern Med. 2014;29(12):1692-701.

11. Concannon TW, Meissner P, Grunbaum JA, McElwee N, Guise J-M, Santa J, et al. A new taxonomy for stakeholder engagement in patient-centered outcomes research. J Gen Intern Med. 2012;27(8):985-91.

12. Ciccarella A, Staley AC, Franco AT. Transforming research: engaging patient advocates at all stages of cancer research. Annals of Translational Medicine. 2018:6(9).

13. Domecq JP, Prutsky G, Elraiya T, Wang Z, Nabhan M, Shippee N, et al. Patient engagement in research: a systematic review. BMC Health Serv Res. 2014:14(89):1-9.

14. Mullins CD, Abdulhalim AM, Lavallee DC. Continuous patient engagement in comparative effectiveness research. JAMA. 2012;207(15):1587-8.

15. Forsythe $L P$, Ellis $L E$, Edmundson L, Sabharwal R, Rein A, Konopka K, et al. Patient and stakeholder engagement in the PCORI pilot projects: description and lessons learned. J Gen Intern Med. 2016;31(1):13-21.

16. Deverka PA, Lavallee DC, Desai PJ, Armstrong J, Gorman M, Hole-Curry L, et al. Facilitating comparative effectiveness research in cancer genomics: evaluating stakeholder perceptions of the engagement process. J Comp Eff Res. 2012;1(4):359-70.

17. Bansal A, Sullivan SD, Hershman DL, Lyman GH, Barlow WE, McCune JS, et al. A stakeholder-informed randomized, controlled comparative effectiveness study of an order prescribing intervention to improve colony stimulating factor use for cancer patients receiving myelosuppressive chemotherapy: the TrACER study. J Comp Eff Res. 2017;6(5):461-70.

18. Ramsey SD, Barlow WE, Gonzalez-Angulo AM, Tunis SR, Baker LH, Crowley J, et al. Integrating comparative effectiveness design elements and endpoints into a phase III, randomized clinical trial (SWOG S1007) evaluating oncotypeDX-guided management for women with breast cancer involving lymph nodes. Contemp Clin Trials. 2013;34(1):1-9.

19. Deverka PA, Bangs R, Kreizenbeck K, Delaney DM, Hershman DL, Blanke CD, et al. A new framework for patient engagement in Cancer clinical trials cooperative group studies. J Natl Cancer Inst. 2018;110(6):553-9.

20. Patient Centered Outcomes Research Institute: Pragmatic Clinical Studies. 2018. https://www.pcori.org/research-results/pragmatic-clinical-studies. Accessed 7 June 2019.

21. SWOG: Our Advocates. 2018. https://www.swog.org/swog-network/ouradvocates. Accessed 7 June 2019.

22. Patient Centered Outcomes Research Institute: Financial Compensation of Patients, Caregivers, and Patient/Caregiver Organizations Engaged in PCORIFunded Research as Engaged Research Partners. 2019. https://www.pcori. org/sites/default/files/PCORI-Compensation-Framework-for-EngagedResearch-Partners.pdf. Accessed 7 June 2019.

\section{Publisher's Note}

Springer Nature remains neutral with regard to jurisdictional claims in published maps and institutional affiliations. 